\title{
Use of dicloxacillin for the isolation and counting of Bifidobacteria from dairy products
}

\author{
T Sozzi 1, P Brigidi 2, O Mignot 1, D Matteuzzi 2* \\ ${ }^{1}$ Nestec SA, Linor, Food Products Development Centre, 1350 Orbe, Switzerland; \\ 2 University of Bologna, Fermentation Chemistry and Industrial Microbiology, Department of Pharma- \\ ceutical Sciences, via Belmeloro 6, 40126 Bologna, Italy
}

(Received 9 May 1990; accepted 5 June 1990)

Summary - The use of the antibiotic dicloxacillin for isolating and counting the Bifidobacteria present in fermented milks is described. The addition of $2 \mu \mathrm{g} / \mathrm{ml}$ of dicloxacillin to TPY medium was found to inhibit the growth of Lactobacilli and Streptococci whereas most Bifidobacteria grew well. TPY medium was found to be more suitable than MRS agar to select Bifidobacteria.

selective media / Bifidobacteria / fermented milk

Résumé - Emploi de la dicloxacilline pour l'isolement et le dénombrement des bifidobactéries dans les produits laitiers. L'utilisation de l'antibiotique dicloxacilline pour isoler et compter les bifidobactéries présentes dans les produits laitiers fermentés est décrite. L'addition de $2 \mu \mathrm{g} / \mathrm{ml}$ de dicloxacilline au milieu TPY s'est révélée efficace pour inhiber totalement la croissance des lactobacilles et des streptocoques, mais par contre sans effet négatif sur la plupart des bifidobactéries. Le milieu TPY s'est révélé plus adéquat que le milieu MRS agar pour la sélection des bifidobactéries.

milieu sélectif / bifidobactérie / lait fermenté

Bifidobacteria are Gram-positive, nonsporing anaerobic rods. Generally of irregular shape, the rods are bifurcated or club-shaped with cell protuberances and branchings (Scardovi, 1986). The typical habitat of this bacterial group is human and animal intestinal tract, where they play a protective role against intestinal infections caused by Salmonella, Shigella and Escherichia coli enteropathogens (Sandine et al, 1972; Poupard et al,
1973). In order to establish a proper identification at genus level, fructose-6- phosphate phosphoketolase, the key enzyme of "bifid shunt" (Lauer and Kandler, 1976) must be determined in cellular extracts. Species identification requires DNA-DNA hybridization (Scardovi, 1986). For the purpose of proper and correct identification, various attempts have been made to obtain a selective medium for isolating $\mathrm{Bi}$ fidobacteria from other intestinal micro-

* Correspondence and reprints 
organisms, especially Streptococci and Lactobacilli. A number of differential media have been suggested based on the addition of several antimicrobial compounds to complex media (Teraguchi et al, 1978; Resnick and Levin, 1981; Munoa and Pares, 1988; Cole and Fuller, 1989; Wijsman et al, 1989). None of these media have proven to be sufficiently selective as all were found to inhibit part of the viable bifidobacterial population.

The problem of properly enumerating Bifidobacteria in dairy products containing Lactobacilli and Streptococci as well as Bifidobacteria themselves, has become an important issue over the last 10 years as Bifidobacteria have become widely employed in preparing fermented milks (Rašić and Kurmann, 1983). The NPLN agar medium proposed by Teraguchi et al (1978), which contains lithium chloride, nalidixic acid, neomycin sulphate and paranomycin sulphate as antimicrobial agents, has, todate, been the most suitable for this purpose. The preparation of this medium is, however, extremely time-consuming and entails the use of 24 components. In consideration of this drawback and given the Bifidobacteria sensitivity to various antimicrobial agents as reported by Sutter and Finegold (1976) and Matteuzzi et al (1983) the authors have screened for an antibiotic capable of being active on the Lactobacilli and on the Streptococci employed in dairy products, especially fermented milks, so as to quickly isolate, identify and count $\mathrm{Bi}$ fidobacteria.

The antimicrobial activity of 30 antibiotics toward the above-mentioned bacteria has been examined using MRS (Merck no 10661) and TPY (Scardovi, 1986) media. The afore-mentioned compounds were examined at $0.5,1.0,2.0$ and $3.0 \mu \mathrm{g} / \mathrm{ml}$ concentrations. Of the tested compounds, only nafcillin and dicloxacillin were found to possess lower MIC (minimal inhibitory concentration) towards Streptococci and Lactobacilli than to Bifidobacteria. Subsequent testing showed that dicloxacillin was more effective than nafcillin for Bifidobacteria selection. Table I shows dicloxacillin MIC values towards 35 strains, mainly of human origin, of Bifidobacteria as well as towards the major species of Lactobacilli and Streptococci used in fermented milks. Clearly then, only 1 out of the 36 Bifidobacteria strains examined are inhibited by dicloxacillin at a concentration of $1 \mu \mathrm{g} / \mathrm{ml}$. For all other strains, the MIC values were equal to or more than $3 \mu \mathrm{g} / \mathrm{ml}$. All strains of lactic acid bacteria of the species Lactobacillus acidophilus, $L$ bulgaricus, $L$ helveticus, Streptococcus thermophilus and $S$ cremoris showed MIC values equal to or less than $1.5 \mu \mathrm{g} / \mathrm{ml}$. Inhibition of the $L$ casei species takes places at concentrations of 2.0 and $2.5 \mu \mathrm{g} / \mathrm{ml}$. Table II illustrates the efficacy of dicloxacillin at a concentration value of 2 $\mu \mathrm{g} / \mathrm{ml}$ in the selection of Bifidobacteria present in some commercial fermented milk samples. It is clear from this table that the use of dicloxacillin allows the identification and the count of the Bifidobacteria present. Other tests conducted by us (data not reported in this paper) have shown that MRS or TPY media added with dicloxacillin at a concentration of $2 \mu \mathrm{g} / \mathrm{ml}$ behave similarly to the NPNL agar medium tested by Teraguchi et al (1978). The latter however, as has already been noted, is particularly difficult and complicated to prepare. In conclusion therefore, it can be stated that the addition of dicloxacillin at a concentration of $2 \mu \mathrm{g} / \mathrm{ml}$ to MRS or, better still, to TPY media can most certainly be recommended for Bifidobacteria isolation and count in fermented milks or dairy products in general. 
Table I. Comparative activities of dicloxacillin against bifidobacteria and some species of lactic acid bacteria.

Activité comparée de la dicloxacilline sur les bifidobactéries et sur quelques espèces de bactéries lactiques.

\begin{tabular}{|c|c|c|c|}
\hline Species & Strains & Source & $\operatorname{MIC}(\mu \mathrm{g} / \mathrm{ml})$ \\
\hline $\begin{array}{l}\text { Bifidobacterium } \\
\text { longum }\end{array}$ & $\begin{array}{l}\text { ATCC } 15707 \\
\text { ATCC } 15708 \\
{ }^{*} \text { B1672-B1678-B2055 } \\
\text { B2407-B2432-B2664 } \\
\text { B2577 }\end{array}$ & $\begin{array}{l}\text { adult intestine } \\
\text { infant intestine } \\
\text { infant intestine }\end{array}$ & $\begin{array}{l}>5 \\
>5 \\
\geq 3\end{array}$ \\
\hline $\begin{array}{l}\text { Bifidobacterium } \\
\text { breve }\end{array}$ & $\begin{array}{l}\text { ATCC } 15700 \\
\text { B622, B626, B632 } \\
\text { B644, B648, B1303 }\end{array}$ & $\begin{array}{l}\text { infant intestine } \\
\text { infant intestine }\end{array}$ & $\begin{array}{l}>5 \\
\geq 4\end{array}$ \\
\hline $\begin{array}{l}\text { Bifidobacterium } \\
\text { bifidum }\end{array}$ & $\begin{array}{l}\text { B1958, B1968 } \\
\text { B2004, B2531 } \\
\text { B2412 }\end{array}$ & $\begin{array}{l}\text { infant intestine } \\
\text { infant intestine }\end{array}$ & $\begin{array}{r}\geq 5 \\
1\end{array}$ \\
\hline $\begin{array}{l}\text { Bifidobacterium } \\
\text { adolescentis }\end{array}$ & $\begin{array}{l}\text { ATCC 15705, ATCC } 15706 \\
{ }^{*} \text { A202-16, A216-21 } \\
\text { A201-3, *115-7 }\end{array}$ & $\begin{array}{l}\text { adult feces } \\
\text { infant feces }\end{array}$ & $\begin{array}{l}3 \\
3\end{array}$ \\
\hline $\begin{array}{l}\text { Bifidobacterium } \\
\text { infantis }\end{array}$ & B1269 & infant feces & 4 \\
\hline $\begin{array}{l}\text { Bifidobacterium } \\
\text { animalis }\end{array}$ & ATCC 27536 & chicken feces & $>5$ \\
\hline $\begin{array}{l}\text { Bifidobacterium } \\
\text { boum }\end{array}$ & ATCC 27917 & cattle rumen & 3 \\
\hline $\begin{array}{l}\text { Bifidobacterium } \\
\text { thermophilum }\end{array}$ & $\begin{array}{l}\text { ATCC25866, "RU294, RU458 } \\
{ }^{*} \text { F333, F454, F466 }\end{array}$ & $\begin{array}{l}\text { cattle rumen } \\
\text { sewage }\end{array}$ & $\begin{array}{l}\geq 4 \\
\geq 4\end{array}$ \\
\hline $\begin{array}{l}\text { Lactobacillus } \\
\text { acidophilus }\end{array}$ & 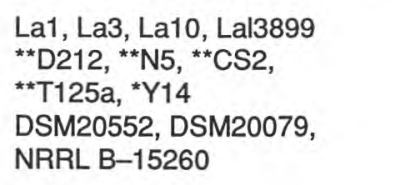 & $\begin{array}{l}\text { dairy products } \\
\text { animal intestine } \\
\text { adult intestine } \\
\text { adult intestine }\end{array}$ & $\begin{array}{r}\leq 1.5 \\
1.0 \\
\leq 1.5 \\
1.0\end{array}$ \\
\hline $\begin{array}{l}\text { Lactobacillus } \\
\text { bulgaricus }\end{array}$ & $\begin{array}{l}\text { YL1, YL2, YL5, YL7 } \\
\text { LB2, LB50 }\end{array}$ & dairy products & 0.5 \\
\hline $\begin{array}{l}\text { Lactobacillus } \\
\text { bulgaricus } \\
\text { "filant" }\end{array}$ & $\begin{array}{l}\text { LFi 1, LFi } 5 \\
\text { LFi12, LFi } 20\end{array}$ & dairy products & 0.5 \\
\hline $\begin{array}{l}\text { Streptococcus } \\
\text { thermophilus }\end{array}$ & $\begin{array}{l}\text { YS1, YS3, YS4, YS7, YS8 } \\
\text { YS10, YS11, YS17, YS20, } \\
\text { YS23, YS27, YS28, YS31 } \\
\text { YS32, YS33, D1 }\end{array}$ & dairy products & $\leq 0.5$ \\
\hline
\end{tabular}




\begin{tabular}{|c|c|c|c|}
\hline $\begin{array}{l}\text { Streptococcus } \\
\text { thermophilus } \\
\text { "filant" }\end{array}$ & $\begin{array}{l}\text { SFi } 3 \text {, SFi } 9, \text { SFi } 12, \\
\text { SFi } 16, \text { SFi } 18, \text { SFi } 19, \\
\text { SFi } 21, \text { SV3, STV } 1 \text {, } \\
\text { STV } 12, \text { STV } 20, \text { STB } 2 \text {, STB } 6\end{array}$ & dairy products & $\leq 1$ \\
\hline $\begin{array}{l}\text { Streptococcus } \\
\text { cremoris }\end{array}$ & STB 1, SCB 9 & dairy products & $\leq 1.5$ \\
\hline $\begin{array}{l}\text { Lactobacillus } \\
\text { casei }\end{array}$ & $\begin{array}{l}\text { ATCC 7469, NCIB 8001, } \\
* * \text { SC2, DSM 20024, DSM } 20011\end{array}$ & - & $\begin{array}{l}2.5 \\
2.0\end{array}$ \\
\hline $\begin{array}{l}\text { Lactobacillus } \\
\text { helveticus }\end{array}$ & $\begin{array}{l}\text { S10.10, S29.9, S30.16 } \\
\text { S30.18, S30.14, S34.17, } \\
\text { S39.4, S40.8 }\end{array}$ & dairy products & 1.0 \\
\hline
\end{tabular}

* From the collection of the Institute of Agricultural Microbiology, University of Bologna, Bologna, Italy. ${ }^{* *}$ From Prof V Bottazzi Faculty of Agriculture, Piacenza - Italy. All other strains derived from International Culture Collections or from our laboratories.

Table II. Presence of bifidobacteria, lactic streptococci and lactobacilli on TPY agar containing 2 $\mu \mathrm{g} / \mathrm{ml}$ dicloxacillin in some commercial fermented milks.

Recherche de bifidobactéries, streptocoques lactiques et lactobacilles dans quelques laits fermentés du commerce sur milieu TPY gélosé contenant $2 \mu \mathrm{g} / \mathrm{ml}$ de dicloxacilline.

\begin{tabular}{llll}
\hline Product & Streptococci & Lactobacilli & Bifidobacteria \\
\hline $\begin{array}{c}\text { Bio-Danone (Gervais } \\
\text { Danone Italiana, Turin) } \\
\text { Kyr (Parmalat, }\end{array}$ & absent & absent & \\
$\begin{array}{l}\text { Parma, Italy) } \\
\text { Latte Natura }\end{array}$ & absent & absent & +++ \\
$\begin{array}{l}\text { (Granarolo, Bologna, } \\
\text { Italy) }\end{array}$ & absent & absent & +++ \\
$\begin{array}{l}\text { Ofilus (Yoplait, } \\
\text { France and Switzerland) }\end{array}$ & absent & absent & +++ \\
$\begin{array}{l}\text { BA (GIE BA, France) } \\
\text { OH (Chambourcy, France) }\end{array}$ & absent & absent & +++ \\
$\begin{array}{l}\text { BI (Laiteries de Savoie, } \\
\text { France) } \\
\text { Yogurt-Bifidus } \\
\text { (Migros, Switzerland) }\end{array}$ & absent & absent & +++ \\
$\begin{array}{l}\text { Fromage-Bifidus } \\
\text { (Migros, Switzerland) }\end{array}$ & absent & absent & +++ \\
\hline
\end{tabular}

+++ : Abundant growth (>1× $\left.10^{5} \mathrm{CFU} / \mathrm{ml}\right)$. 


\section{REFERENCES}

Cole CB, Fuller R (1989) Enumeration of intestinal Bifidobacteria by growth on a semiselective medium and GLC assay of acetic acid production. Microb Ecol Health Dis 2, 227-230

Lauer E, Kandler O (1976) Mechanismus der Variation der Verhältnisses Acetat/Lactat bei der Vergärung von Glucose durch Bifidobakterien. Arch Mikrobiol 110, 271-277

Matteuzzi D, Crociani F, Brigidi P (1983) Antimicrobial susceptibility of Bifidobacterium. Ann Microbiol (Inst Past) 134A, 339-349

Manoa FJ, Pares R (1988) Selective medium for isolation and enumeration of Bifidobacterium spp. Appl Environ Microbiol 54, 1715-1718

Poupard JA, Husain I, Norris RF (1973) Biology of Bifidobacteria. Bacteriol Rev 37, 136-165

Rasic JL, Kurmann JA (1983) Bifidobacteria and Their Role. Birklauser Verlag, Basel

Resnik IG, Levin MA (1981) Quantitative procedure for enumeration of Bifidobacteria. Appl Environ Microbiol 42, 427-432
Sandine WE, Muralidara KS, Elliker PR, England DC (1972) Lactic acid bacteria in food and health: a review with special reference to enteropathogenic Escherichia coli as well as certain enteric diseases and their treatment with antibiotics and lactobacilli. J Milk Food Technol 35, 691-702

Scardovi V (1986) The genus Bifidobacterium. In: Bergey's Manual of Systematic Bacteriology (Sneath PHA, Sharpe ME, Holt JG, eds) The Williams and Wilkins Co, Baltimore 2, 1418-1434

Sutter VL, Finegold SM (1976) Susceptibility of anaerobic bacteria to 23 antimicrobial agents. Antimicrob Agents Chemother 10, 736-752

Teraguchi S, Uehara M, Ogasa K, Mitsuoka T (1978) Enumeration of Bifidobacteria in dairy products. Jpn J Bacterio/ 33, 753-761

Wijsman MR, Hereijgers JLPM, Groote JMFH (1989) Selective enumeration of Bifidobacteria in fermented dairy products. Neth Milk Dairy J 43, 395-405 